\title{
Atención de pacientes con errores innatos del metabolismo durante la pandemia de COVID-19 en México: importancia de la telemedicina
}

\section{Care of patients with inborn errors of metabolism during the COVID-19 pandemic in Mexico: importance of telemedicine}

Sara Guillén-López, Lizbeth López-Mejía, Rosa Itzel Carrillo-Nieto, Isabel Ibarra-González, Leticia BelmontMartínez, Marcela Vela-Amieva

\begin{abstract}
Resumen
OBJETIVO: Comunicar los desenlaces de la experiencia con la aplicación de algunas herramientas básicas de telemedicina para la atención de pacientes con errores innatos del metabolismo durante la pandemia de COVID-19 y describir la atención presencial llevada a cabo en el mismo periodo, en un centro pediátrico de tercer nivel de atención. MATERIALES Y MÉTODOS: Estudio retrospectivo de pacientes con errores innatos del metabolismo que recibieron atención presencial o mediante herramientas remotas (correos electrónicos o mensajería instantánea por Whats App ${ }^{\circledR}$ ) en el Laboratorio de Errores Innatos del Metabolismo y Tamiz del Instituto Nacional de Pediatría, del 23 de marzo al 23 de julio del 2020.

RESULTADOS: Se atendieron 117 pacientes con 26 diferentes errores innatos del metabolismo. Se otorgaron 97 consultas presenciales y se enviaron 308 correos electrónicos y 137 mensajes telefónicos. Solo se registró una descompensación metabólica severa en un paciente con acidemia propiónica, que requirió internamiento urgente. No ocurrió ninguna defunción. Siete pacientes fueron de primera vez, 4 de ellos llegaron por tener tamiz neonatal anormal; todos llegaron tardíamente (4-9 meses de edad). Los principales obstáculos detectados para brindar la atención mediante telemedicina fueron la carencia de la plataforma informática respectiva en la institución y de computadoras con internet por parte de los pacientes.

CONCLUSIÓN: Los desenlaces señalan las ventajas de algunas de las herramientas de la telemedicina y revelan la necesidad de contar con una plataforma moderna de telesalud institucional que permita atender e instruir a los pacientes y capacitar al personal de salud implicado en la atención de enfermos con algún error innato del metabolismo. PALABRAS CLAVE: Telemedicina; errores innatos del metabolismo; errores innatos del metabolismo de los aminoácidos; acidemias orgánicas; SARS-CoV-2; tamiz metabólico; tamiz neonatal.
\end{abstract}

Abstract

OBJECTIVE: To communicate the outcomes of the experience with the application of some basic telemedicine tools in the care of patients with inborn errors of metabolism during the COVID-19 pandemic and to describe the face-to-face care carried out in the same period, in a third level of care pediatric center.

MATERIALS AND METHODS: Retrospective study of patients with inborn errors of metabolism who received face-to-face or remote care (e-mail or instant messaging via Whats $\mathrm{App}^{\circledR}$ ) at the National Institute of Pediatrics Inborn Errors of Metabolism and Screening Laboratory, March 23-July 23, 2020.

RESULTS: 117 patients with 26 different IEM were treated. 97 outpatient consultations were given, and 308 emails and 137 telephone messages were sent. Severe metabolic decompensation was only observed in one propionic acidemia patient, who required urgent admission. No deaths were registered. Seven patients were seen for the first time and 4 of them were referred due to an abnormal newborn screening result, but
Laboratorio de Errores Innatos del Metabolismo y Tamiz, Instituto Nacional de Pediatría, Ciudad de México.

Recibido: 18 de agosto de 2020

Aceptado: 23 de noviembre de 2020

Correspondencia

Marcela Vela-Amieva

dravelaamieva@yahoo.com

Este artículo debe citarse como: GuiIlén-López S, López-Mejía L, CarrilloNieto RI, Ibarra-González I, BelmontMartínez L, Vela-Amieva M. Atención de pacientes con errores innatos del metabolismo durante la pandemia de COVID-19 en México: importancia de la telemedicina. Acta Pediatr Méx 2021; 42 (1): 11-20. 
all these patients came late at ages between 4-9 months old. The main obstacles identified to provide telemedicine care were the lack of a modern telehealth platform in our institution and the lack of computers with internet by patients.

CONCLUSION: Our results point out benefits of some telemedicine strategies but reveal the need for a modern institutional telehealth platform that allows the medical care for IEM patients and the training of both patients and health personnel involved with them. KEY WORDS: Telemedicine; Metabolism, inborn errors; Amino acid metabolism, inborn errors; Organic acidemias; SARS-CoV-2; Metabolic screening; Neonatal screening.

\section{ANTECEDENTES}

La telesalud, entendida como la práctica de la medicina a distancia, tiene una larga historia, pero su expansión y mayor uso ocurrió en el decenio de 1980, gracias a la llegada de las comunicaciones digitales y a las computadoras de bajo costo. ${ }^{1}$ La telemedicina se define como el uso de la tecnología electrónica o de los medios de comunicación, con el fin de diagnosticar o tratar a un paciente, y proporcionar servicios de seguimiento remoto o consultar con otros proveedores de atención médica con respecto al diagnóstico, tratamiento o evolución clínica de un paciente. ${ }^{2}$

A partir de diciembre de 2019, desafortunadamente, el mundo se ha visto afectado por la pandemia del virus SARS-CoV-2, agente causal de la enfermedad denominada COVID-19. En México, esta pandemia había provocado, hasta noviembre de 2020, poco más de 100,000 muertes e infectado a miles de personas. ${ }^{3}$ En muchos países, a lo largo de la pandemia de SARS-CoV-2, la telemedicina ha sido una alternativa para seguir brindando la atención en muchos campos de la Medicina, incluida la Pediatría. ${ }^{4,5}$

Los errores innatos del metabolismo son un grupo complejo de enfermedades genéticas, casi siempre multisistémicas, que suelen tener una evolución clínica crónica y pueden asociarse con descompensaciones metabólicas agudas; si bien los errores innatos del metabolismo pueden manifestarse a cualquier edad, su inicio es predominante en los primeros años de la vida. ${ }^{6}$ Lo común es que sean causados por la pérdida parcial o total de una enzima, cofactor, transportador o una proteína auxiliar del metabolismo y casi siempre tienen un patrón de herencia autosómico recesivo o ligado al cromosoma $X .{ }^{6,7}$ Una característica sobresaliente de los errores innatos del metabolismo es que se trata de enfermedades individualmente raras, pero que colectivamente repercuten de manera importante en la morbilidad y mortalidad infantil. Sin embargo, por ser de baja prevalencia, el personal de salud de primer contacto en las unidades médicas no siempre tiene la capacitación suficiente para sospecharlas ni para atenderlas; por esto es necesario el apoyo de los centros de diagnóstico y tratamiento especializados. ${ }^{7}$

En el Laboratorio de Errores Innatos del Metabolismo y Tamiz del Instituto Nacional de Pediatría se atienden pacientes con trastornos genéticos poco comunes, sobre todo aminoacidopatías, acidemias orgánicas y defectos de la beta-oxidación de los ácidos grasos, ${ }^{8}$ desde su diagnóstico bioquímico y molecular, hasta su tratamiento médico y nutricional. ${ }^{9,10,11}$ Cada año se dan más de 500 consultas presenciales. ${ }^{12}$ Los pacientes suelen ser referidos de dos formas: a) los que tienen un estudio de tamiz neonatal sospechoso practicado en otro laboratorio, y b) los que tienen síntomas clínicos sugerentes y cuyos médicos tratantes sospechan un error innato del metabolismo. La cantidad anual de pacientes hospitalizados en el Instituto Nacional de Pediatría, con un error innato del metabolismo, es cercana a $33 .{ }^{13}$

Los errores innatos del metabolismo, al igual que otras enfermedades crónicas de la infancia, 
requieren vigilancia constante, sumamente especializada. El sistema de telesalud es una alternativa para mejorar su atención, sobre todo en quienes la nutrición es el punto central del tratamiento. ${ }^{14}$ En México aún no existe un marco normativo claro para brindar servicios de Telesalud; sin embargo, el Centro Nacional de Excelencia Tecnológica en Salud (CENETEC) ha emitido una serie de documentos que compilan el marco jurídico aplicable en la materia, entre los que destacan el Reglamento de la Ley General de Salud en materia de prestación de servicios de atención médica y la Norma Oficial Mexicana del expediente clínico. ${ }^{15-18}$

Durante los primeros meses de la pandemia de COVID-19 ha existido una gran revaloración de la telemedicina y la cantidad de trabajos publicados entre enero y septiembre de 2020 sobre el tema rebasan 4000 publicaciones indizadas en el buscador PubMed (https://pubmed.ncbi.nlm.nih. gov/?term=telemedicine\&filter=datesearch.y_ 1 \& sort=date). En este contexto, nuestro grupo hizo una serie de adaptaciones de atención mediante el uso de herramientas de telemedicina.

El objetivo de este estudio fue: comunicar los desenlaces de la experiencia con el uso de algunas herramientas básicas de telemedicina en la atención de pacientes con errores innatos del metabolismo durante la pandemia de COVID-19 y describir la atención presencial Ilevada a cabo en el mismo periodo, en un centro pediátrico de tercer nivel de atención.

\section{MATERIALES Y MÉTODOS}

Estudio retrospectivo de pacientes con errores innatos del metabolismo que recibieron atención presencial o mediante herramientas remotas (correos electrónicos o mensajería instantánea por Whats $\mathrm{App}^{\circledR}$ ) en el Laboratorio de Errores Innatos del Metabolismo y Tamiz del Instituto Nacional de Pediatría, del 23 de marzo al 23 de julio del 2020 (cuatro meses).
Las fuentes de información utilizadas fueron: expedientes electrónicos, hojas oficiales de consulta diaria del médico, agenda estadística institucional del $2018^{12}$ y las bases de Excel en las que se consignaron todos los tipos de atención remota brindados, los datos demográficos, la distancia promedio en $\mathrm{km}$ desde el lugar de origen hasta el centro de tratamiento en la Ciudad de México. Durante este periodo, un pediatra y dos nutriólogas especialistas en errores innatos del metabolismo brindaron la atención presencial y en línea, con el apoyo de otros dos pediatras en línea, dos químicos y dos técnicos de laboratorio. También se registraron los principales obstáculos para la comunicación por vía electrónica.

Por último, se llevó a cabo una entrevista semiestructurada al personal de salud clave que trabaja en el laboratorio $(n=7)$ con el fin de identificar cuáles eran las principales barreras para la atención médico-nutricional en pacientes con errores innatos del metabolismo. Se les hizo la siguiente pregunta: ¿Cuál o cuáles considera usted que son los mayores obstáculos para la atención médiconutricional en telemedicina en la institución?

\section{RESULTADOS}

\section{Atención médica presencial}

Del 23 de marzo al 23 de julio de 2020 se atendieron 117 pacientes con 26 diferentes errores innatos del metabolismo (Cuadro 1); de ellos, 110 eran ya conocidos por el servicio antes de la pandemia COVID-19 y 7 fueron casos de primera vez en quienes el diagnóstico se confirmó en el laboratorio mediante cromatografía de líquidos de alta resolución, espectrometría de masas en tándem y cromatografía de gases acoplada a espectrometría de masas. Todos los pacientes de primera vez fueron atendidos en forma presencial. Las especificaciones de la forma en que llegaron al servicio y su cuadro clínico inicial se detallan en el Cuadro 2. 
Cuadro 1. Diagnósticos de los 117 pacientes atendidos durante el periodo de estudio, con sus respectivos diagnósticos metabólicos

\begin{tabular}{|c|c|}
\hline Diagnóstico & $\begin{array}{c}\text { Cantidad de pacientes } \\
\text { y porcentaje }\end{array}$ \\
\hline $\begin{array}{l}\text { Fenilcetonuria (deficiencia de } \\
\text { fenilalanina hidroxilasa) y otras } \\
\text { hiperfenilalaninemias (deficien- } \\
\text { cia del metabolismo de la } \mathrm{BH} 4 \text { ) }\end{array}$ & $36(30.8 \%)$ \\
\hline $\begin{array}{l}\text { Enfermedad de orina de jarabe } \\
\text { de arce (maple) }\end{array}$ & $10(8.5 \%)$ \\
\hline Cistinosis & $10(8.5 \%)$ \\
\hline Acidemia metilmalónica & $9 \quad(7.7 \%)$ \\
\hline $\begin{array}{l}\text { Acidemia 3-hidroxi-3 } \\
\text { metilglutárica }\end{array}$ & $7 \quad(6 \%)$ \\
\hline Defectos de cobalamina & $6 \quad(5.1 \%)$ \\
\hline Acidemia isovalérica & $4 \quad(3.4 \%)$ \\
\hline Acidemia propiónica & $4 *(3.4 \%)$ \\
\hline Citrulinemia & $4 \quad(3.4 \%)$ \\
\hline Argininemia & $3 \quad(2.6 \%)$ \\
\hline $\begin{array}{l}\text { Deficiencia de } \\
\text { 3-metilcrotonil-CoA carboxilasa }\end{array}$ & $3(2.6 \%)$ \\
\hline Tirosinemia tipo 1 & $3 \quad(2.6 \%)$ \\
\hline Acidemia arginosuccínica & $2(1.7 \%)$ \\
\hline Acidemia glutárica tipo 2 & $2(1.7 \%)$ \\
\hline Deficiencia de OTC & $2(1.7 \%)$ \\
\hline Galactosemia & $2(1.7 \%)$ \\
\hline Aciduria 3-metilglutacónica & $1 \quad(0.85 \%)$ \\
\hline Atrofia girata & $1(0.85 \%)$ \\
\hline Deficiencia de biotinidasa & $1 \quad(0.85 \%)$ \\
\hline $\begin{array}{l}\text { Deficiencia de carnitina } \\
\text { palmitoiltransferasa I }\end{array}$ & $1 \quad(0.85 \%)$ \\
\hline $\begin{array}{l}\text { Deficiencia de hidroxiacil-CoA } \\
\text { deshidrogenasa de cadena larga }\end{array}$ & $1 \quad(0.85 \%)$ \\
\hline $\begin{array}{l}\text { Deficiencia múltiple de } \\
\text { carboxilasas }\end{array}$ & $1 \quad(0.85 \%)$ \\
\hline Glucogenosis III & $1 \quad(0.85 \%)$ \\
\hline Homocistinuria & $1 \quad(0.85 \%)$ \\
\hline Síndrome $\mathrm{HHH}$ & $1 \quad(0.85 \%)$ \\
\hline Tirosinemia tipo 2 & $1 \quad(0.85 \%)$ \\
\hline
\end{tabular}

* Paciente con descompensación metabólica que requirió internamiento en Urgencias.
En los cuatro meses del estudio se atendieron 97 consultas presenciales: 70 en consulta externa y 27 en hospitalización, a 42 pacientes diferentes. Del total de consultas otorgadas 50\% requirieron un cambio en el control nutricional, a $8 \%$ se les modificó el tratamiento farmacológico y $39 \%$ acudieron a la institución a recoger una receta por alimento médico metabólico.

\section{Consultas y orientación por medios electrónicos}

Durante el periodo de estudio se enviaron 308 correos electrónicos y respondieron 137 mensajes electrónicos instantáneos. Las causas están desglosadas en el Cuadro 3. El lugar de procedencia de los pacientes, la cantidad de mensajes electrónicos y la distancia desde el lugar de procedencia se muestran en el Cuadro 4.

\section{Evolución de los pacientes con errores innatos del metabolismo}

Los pacientes ya conocidos permanecieron estables, en sus domicilios, y solo se registró una descompensación metabólica severa en un paciente con acidemia propiónica. Los datos de alarma (vómito, rechazo al alimento y somnolencia) los notificó la madre a través de un mensaje electrónico y una videollamada, en la que se observaron: palidez, hipoactividad y decaimiento. Se le indicó que acudiera de inmediato al servicio de Urgencias en donde se documentaron: acidosis metabólica e hiperamonemia, motivo por el que permaneció hospitalizado durante 11 días, al cabo de los cuales fue dado de alta a su domicilio, por mejoría. En este periodo no se registraron defunciones.

\section{Pacientes con errores innatos del metabolismo y COVID-19}

Solo se documentó el caso de un niño con enfermedad de orina de jarabe de arce (maple) 
Guillén-López S, et al. Pacientes con errores innatos del metabolismo en la pandemia COVID-19

INP

Cuadro 2. Pacientes de primera vez, forma de referencia y principales características clínicas al momento del diagnóstico

\begin{tabular}{|c|c|c|}
\hline $\begin{array}{l}\text { Tipo de error innato del meta- } \\
\text { bolismo }\end{array}$ & Referencia a nuestro servicio & $\begin{array}{l}\text { Principales características clínicas al momento } \\
\text { del diagnóstico }\end{array}$ \\
\hline $\begin{array}{l}\text { Enfermedad de orina de jarabe } \\
\text { de arce (maple) }\end{array}$ & $\begin{array}{l}\text { Interconsulta en el servicio de Neo- } \\
\text { natología de nuestra institución. }\end{array}$ & $\begin{array}{l}\text { Recién nacido de } 20 \text { días de vida, con deterioro } \\
\text { neurológico súbito, crisis convulsivas, vómito, re- } \\
\text { chazo al alimento, apneas, acidosis metabólica e } \\
\text { hiperamonemia. }\end{array}$ \\
\hline Aciduria glutárica tipo 2 & $\begin{array}{l}\text { Interconsulta en el servicio de Neo- } \\
\text { natología de nuestra institución }\end{array}$ & $\begin{array}{l}\text { Recién nacido de } 17 \text { días de vida, con antecedente } \\
\text { de prematurez las } 32 \text { semanas, crisis convulsivas, } \\
\text { hipoglucemia neonatal, hiperlactatemia, hiperamo- } \\
\text { nemia y acidosis metabólica. }\end{array}$ \\
\hline $\begin{array}{l}\text { Deficiencia de } \\
\text { ornitinatranscarbamilasa }\end{array}$ & $\begin{array}{l}\text { Interconsulta en el servicio de Gas- } \\
\text { tronutrición de nuestra institución }\end{array}$ & $\begin{array}{l}\text { Niño de } 1 \text { año de edad que inició con evacuaciones } \\
\text { disminuidas en consistencia, vómito, rechazo al } \\
\text { alimento, somnolencia, alteración en la marcha, e } \\
\text { hiperamonemia. }\end{array}$ \\
\hline $\begin{array}{l}\text { Deficiencia de } \\
\text { 3-metilcrotonil-CoA carboxilasa }\end{array}$ & $\begin{array}{l}\text { Referido por un pediatra externo, por } \\
\text { tamiz neonatal anormal }\end{array}$ & Niña de 5 meses de edad, asintomática. \\
\hline Hiperfenilalaninemia & $\begin{array}{l}\text { Referido de una unidad de salud de } \\
\text { primer nivel de atención, por tamiz } \\
\text { neonatal anormal }\end{array}$ & Niña de 8 meses de edad, asintomática. \\
\hline $\begin{array}{l}\text { Acidemia metilmalónica por } \\
\text { defecto del metabolismo de la } \\
\text { cobalamina }\end{array}$ & $\begin{array}{l}\text { Referido por un pediatra externo, por } \\
\text { tamiz neonatal anormal }\end{array}$ & $\begin{array}{l}\text { Niño de } 9 \text { meses de edad, con hipotonía, retraso en } \\
\text { hitos del neurodesarrollo, somnolencia, hiperamo- } \\
\text { nemia, acidosis metabólica con anión GAP elevado. } \\
\text { Antecedente de sepsis de foco pulmonar. }\end{array}$ \\
\hline $\begin{array}{l}\text { Hiperfenilalaninemia por } \\
\text { defecto de la } \mathrm{BH} 4 \text { (tetrahidro- } \\
\text { biopterina) }\end{array}$ & $\begin{array}{l}\text { Referido por un pediatra externo, por } \\
\text { tamiz neonatal anormal }\end{array}$ & $\begin{array}{l}\text { Niña de } 4 \text { meses con distonía, piel y pelo claros, re- } \\
\text { traso en hitos del desarrollo, sialorrea y movimientos } \\
\text { oculares anormales. }\end{array}$ \\
\hline
\end{tabular}

Nota: todos los pacientes de nuevo diagnóstico se atendieron de manera presencial.

Cuadro 3. Distancia máxima del lugar de procedencia de los pacientes $(n=117)$ y cantidad de consultas presenciales y mensajes electrónicos enviados (continúa en la siguiente página)

\begin{tabular}{|c|c|c|c|c|}
\hline Lugar de procedencia & Cantidad de pacientes & $\begin{array}{l}\text { Distancia máxima del lugar de } \\
\text { procedencia a nuestro centro } \\
\text { médico }(\mathrm{km})\end{array}$ & Presencial & Electrónico \\
\hline Ciudad de México & 30 & 22 & 13 & 17 \\
\hline Morelos & 2 & 72 & 2 & 0 \\
\hline Estado de México & 20 & 117 & 11 & 9 \\
\hline Tlaxcala & 2 & 125 & 1 & 1 \\
\hline Puebla & 7 & 137 & 4 & 3 \\
\hline Hidalgo & 7 & 141 & 2 & 5 \\
\hline Querétaro & 4 & 225 & 1 & 3 \\
\hline Michoacán & 1 & 296 & 0 & 1 \\
\hline Guerrero & 5 & 364 & 3 & 2 \\
\hline Guanajuato & 4 & 390 & 0 & 4 \\
\hline Veracruz & 4 & 411 & 0 & 4 \\
\hline San Luis Potosí & 3 & 425 & 0 & 3 \\
\hline
\end{tabular}


Cuadro 3. Distancia máxima del lugar de procedencia de los pacientes $(n=117)$ y cantidad de consultas presenciales y mensajes electrónicos enviados (continuación)

\begin{tabular}{|c|c|c|c|c|}
\hline Lugar de procedencia & Cantidad de pacientes & $\begin{array}{l}\text { Distancia máxima del lugar de } \\
\text { procedencia a nuestro centro } \\
\text { médico }(\mathrm{km})\end{array}$ & Presencial & Electrónico \\
\hline Oaxaca & 3 & 467 & 1 & 2 \\
\hline Aguascalientes & 1 & 505 & 0 & 1 \\
\hline Jalisco & 7 & 535 & 3 & 4 \\
\hline Colima & 1 & 723 & 0 & 1 \\
\hline Tabasco & 3 & 765 & 1 & 2 \\
\hline Durango & 2 & 897 & 0 & 2 \\
\hline Monterrey & 1 & 920 & 0 & 1 \\
\hline Sinaloa & 2 & 1218 & 0 & 2 \\
\hline Yucatán & 1 & 1324 & 0 & 1 \\
\hline Chihuahua & 3 & 1440 & 0 & 3 \\
\hline Baja California Sur & 3 & 1680 & 0 & 3 \\
\hline Baja California & 1 & 2700 & 0 & 1 \\
\hline
\end{tabular}

Cuadro 4. Motivos que originaron la consulta por medios electrónicos

\begin{tabular}{|c|c|c|c|}
\hline Motivo de la consulta & Correo electrónico & Mensajería instantánea & Total \\
\hline Ajuste de tratamiento nutricional & 187 & 6 & 193 \\
\hline $\begin{array}{l}\text { Solicitud de información administrativa } \\
\text { (programación de cita y solicitud de valoración) }\end{array}$ & 94 & 34 & 128 \\
\hline Pregunta sobre el tratamiento o evolución o seguimiento médico & 0 & 92 & 92 \\
\hline $\begin{array}{l}\text { Consulta de reporte de laboratorio } \\
\text { (vigilancia del padecimiento) }\end{array}$ & 17 & 5 & 22 \\
\hline Ajuste de tratamiento farmacológico & 10 & 0 & 10 \\
\hline Total & 308 & 137 & 445 \\
\hline
\end{tabular}

que tuvo prueba molecular positiva para SARSCoV-2, y que fue totalmente asintomático. La prueba se le indicó debido a que su padre tuvo neumonía que ameritó hospitalización y, como parte de la vigilancia epidemiológica, se les practicó la prueba a todos los miembros de la familia, ${ }^{4}$ resultando todos positivos. A este paciente se le dio orientación electrónica durante 14 días. Desde luego que no hay certeza de cuántos pacientes estuvieron infectados por SARS-CoV-2, puesto que a muy pocos se les hizo la prueba diagnóstica.
Principales obstáculos percibidos por el personal de salud para la atención médica de errores innatos del metabolismo por telemedicina en México

Los principales obstáculos percibidos se muestran en el Cuadro 5.

\section{DISCUSIÓN}

La pandemia de COVID-19 ha forzado a muchos pacientes con enfermedades crónicas a 
permanecer en casa y posponer sus consultas presenciales de seguimiento; ${ }^{19}$ pero al mismo tiempo ha constituido una oportunidad sin precedente para utilizar las herramientas de la telemedicina, incluidos los mensajes electrónicos, correos o videollamadas, con el propósito de alcanzar las metas de la atención médica, consistentes en mejorar la salud de la población, prevenir daños y complicaciones, reducir los costos de la atención y mejorar la integración médico-paciente. ${ }^{20,21}$

Uno de los objetivos del tratamiento de pacientes con errores innatos del metabolismo es evitar que sufran descompensaciones, que generalmente son graves, requieran internamientos en los servicios de Urgencias que, a veces, ponen en riesgo la vida o complican la evolución. ${ }^{13}$ Para evitar esas descompensaciones deben practicar-

Cuadro 5. Principales obstáculos detectados por el personal para brindar atención por telemedicina a las familias o responsables de los pacientes con enfermedad innata del metabolismo

\begin{tabular}{|c|c|}
\hline Obstáculo detectado & $\begin{array}{l}\text { Número de } \\
\text { respuestas } \\
\text { del personal }\end{array}$ \\
\hline \multicolumn{2}{|l|}{ Inherentes a la institución } \\
\hline $\begin{array}{l}\text { Programa incipiente de telemedicina en } \\
\text { nuestra institución }\end{array}$ & $7 / 7$ \\
\hline \multicolumn{2}{|l|}{ Inherentes a los pacientes } \\
\hline $\begin{array}{l}\text { Algunos pacientes carecen o no tienen } \\
\text { acceso a computadora o teléfono celular o } \\
\text { falta de señal satelital, e internet en lugares } \\
\text { remotos en donde viven los pacientes }\end{array}$ & $7 / 7$ \\
\hline $\begin{array}{l}\text { Dificultades para comprender el tratamien- } \\
\text { to nutricional o farmacológico }\end{array}$ & $7 / 7$ \\
\hline $\begin{array}{l}\text { Falta de conocimientos para el manejo } \\
\text { de la tecnología de la información y la } \\
\text { comunicación }\end{array}$ & $7 / 7$ \\
\hline $\begin{array}{l}\text { Dificultades para expresar sus necesidades } \\
\text { de forma clara. }\end{array}$ & $6 / 7$ \\
\hline $\begin{array}{l}\text { Cambio constante de teléfonos móviles de } \\
\text { los padres (principalmente por robo) }\end{array}$ & $4 / 7$ \\
\hline $\begin{array}{l}\text { Falta de precisión en los datos completos } \\
\text { para realizar las valoraciones nutricionales } \\
\text { (peso, talla, recordatorio de alimentos, } \\
\text { resultados de estudios de laboratorio) }\end{array}$ & $4 / 7$ \\
\hline
\end{tabular}

se cuantificaciones periódicas de aminoácidos y acilcarnitinas y un constante ajuste de medicamentos y del tratamiento dietético. Esto implica tener una comunicación permanente con los padres o cuidadores, y acudir con frecuencia al centro de tratamiento. ${ }^{6,22}$

Durante los primeros cuatro meses de la pandemia en nuestro centro se atendieron 117 pacientes con 26 diagnósticos distintos; el más frecuente fue la fenilcetonuria y otras hiperfenilalaninemias, incluidos los defectos del metabolismo de la tetrahidrobiopterina (30.8\%), seguida de pacientes con enfermedad de orina de jarabe de maple (8.5\%), cistinosis $(8.5 \%)$ y acidemia metilmalónica (7.7\%). Esta casuística es similar a la reportada previamente por nuestro grupo. ${ }^{23}$ Solo ocurrió una descompensación metabólica en un paciente con acidemia propiónica que requirió hospitalización urgente (Cuadro 1) y cuyos datos de alarma se advirtieron gracias a la telemedicina (mensajes telefónicos y videollamada). Esta baja proporción de descompensaciones $(0.85 \%)$ puede tener varias explicaciones:

Los pacientes permanecieron resguardados en casa y el control de su tratamiento nutricional se cumplió de manera más estricta por los padres, con lo que se evitaron las transgresiones nutricionales.

Al permanecer resguardados, también disminuyó la posibilidad de contraer otras infecciones respiratorias, que se sabe son una de las primeras causas de descompensación metabólica. ${ }^{13}$

La orientación y el acompañamiento constante que recibieron mediante las herramientas básicas de telemedicina (correos y mensajes electrónicos) permitieron hacer los ajustes constantes del tratamiento nutricional requeridos para mantener su buen estado de salud. Este último punto es similar a lo encontrado por otros autores, pero no en la circunstancia de la pandemia de 
COVID-19, ${ }^{24,25,26}$ lo que hace nuestro trabajo original.

Un desenlace interesante de este estudio fue que, a pesar de la pandemia, los diagnósticos de errores innatos del metabolismo se siguieron estableciendo y que los pacientes de primera vez llegaron de dos maneras distintas: mediante las interconsultas de otros servicios de la institución (3 de 7, 43\%), sobre todo del servicio de Neonatología y por referencia de otros médicos u otros centros de salud debido a un reporte anormal de tamiz neonatal (4 de 7; 57\%). Cuadro 2

Los pacientes referidos por tamiz neonatal anormal Ilegaron muy tardíamente, entre los 4 y 9 meses de edad y, al menos dos de ellos, ya con síntomas ostentosos de la enfermedad (Cuadro 2). Esta observación lleva a replantear la necesidad de reforzar la capacitación para los programas de tamiz neonatal en México que, si bien han avanzado en la parte preanalítica y analítica, ${ }^{27}$ tienen notorias fallas de seguimiento postanalítico, sobre todo en lo referente a la confirmación expedita de los casos y al inicio oportuno del tratamiento. Además, los programas debidamente estructurados de tamiz neonatal deben tener planes de contingencia para seguir funcionando y evitar el retraso en la confirmación diagnóstica e inicio temprano del tratamiento. ${ }^{28}$

El grupo de pacientes analizados comprendió a individuos de todo el país que, en ocasiones, tienen que recorrer grandes distancias para acudir a sus consultas de seguimiento. En el Cuadro 3 se muestra que los pacientes procedían de 24 entidades federativas distintas, algunas de ellas a más de $2700 \mathrm{~km}$ de distancia. Sin embargo, a pesar de la lejanía, algunos pudieron recibir orientación durante la pandemia, al menos por vía electrónica, lo que quizá favoreció el control del tratamiento y la prevención de descompensaciones. Además, la enorme distancia que tienen que recorrer muchos de los pacientes para acudir a nuestro centro, también señala la necesidad de desarrollar clínicas locales especializadas en el estudio y tratamiento de errores innatos del metabolismo, y la telemedicina puede ser una solución para contribuir a la capacitación del personal de salud y al desarrollo de esos centros.

Al igual que otros autores que establecen la utilidad de la telesalud, incluidos la atención telefónica, videos o mensajes para mejorar la atención en tiempo real de los pacientes, ${ }^{14,19,20}$ nuestros resultados apoyan el uso de la telemedicina como una alternativa para ofrecer una atención continua a los pacientes con enfermedades innatas del metabolismo, puesto que puede mejorar el seguimiento médico y nutricional y, por lo tanto, conduce a mejor control de la enfermedad. Las causas que motivaron la consulta por medios electrónicos se señalan en el Cuadro 4; destaca que el primer motivo fue el ajuste nutricional (193 de 445), seguido de solicitudes de información administrativa, como la programación de citas (128 de 445).

En el Cuadro 5 se enlistan los principales obstáculos identificados por el personal de salud de nuestro servicio para brindar la atención mediante telemedicina; los más mencionados fueron: la falta de una plataforma moderna de telemedicina en la institución y la carencia o falta de acceso a computadoras con internet por parte de los pacientes. Es importante capacitar a los cuidadores para que aprendan a utilizar adecuadamente los recursos tecnológicos para consultas remotas, para que llenen debidamente los recordatorios nutricionales y realicen correctamente las mediciones antropométricas requeridas para la consulta (peso y talla). Otra alternativa es contar con un grupo de apoyo que incluya, al menos, un médico y un nutriólogo en cada comunidad para tener consultas médicas y nutricionales mediante telemedicina.

Las consultas virtuales no son un sustituto de las visitas presenciales porque una gran limitante 
es la falta de la exploración física; sin embargo, la telemedicina es una herramienta útil que facilita la comunicación entre los pacientes y los profesionales de la salud, ofrece información y cambios en el tratamiento de forma oportuna para evitar complicaciones graves y mejorar la calidad de vida de los pacientes.

\section{CONCLUSIONES}

Lo aquí informado pone de manifiesto los beneficios de algunas estrategias de telemedicina y revelan la necesidad de contar con una plataforma de telesalud institucional que cumpla con todos los criterios para la calidad de la atención en salud y favorezca la confianza del paciente y del profesional de la salud para dar y recibir atención médica a distancia de calidad, con apego al marco normativo vigente.

\section{Agradecimientos}

A la QFB Aída Jannet Hernández Montiel y al técnico. Luis Ricardo Morales González, por la realización de los estudios de laboratorio metabólico durante la pandemia COVID-19.

\section{REFERENCIAS}

1. Global diffusion of eHealth: making universal health coverage achievable. Report of the third global survey on eHealth. Geneva: World Health Organization; 2016. Licence: CC BY-NC-SA 3.0 IGO.

2. Wijesooriya NR, et al. COVID-19 and telehealth, education, and research adaptations [published online ahead of print, 2020 Jun 18]. Paediatr Respir Rev. 2020; S1526-0542 (20): 30094-4. doi.10.1016/j.prrv.2020.06.009.

3. Secretaría de Salud, información general CoVID-10, México. https://coronavirus.gob.mx/datos/.

4. Hollander JE, Carr BG. Virtually perfect? Telemedicine for Covid-19. N Engl J Med 2020; 382 (18): 1679-81. Epub 2020/03/12.

5. Chandler AL, et al. Telemedicine in pediatrics: possibilities and pitfalls. Pediatr Rev. 2020; 41 (7): 376-378. doi.10.1542/pir.2019-0171.

6. Hoffmann GF, Zschocke J, Nyhan WL, Eds. Inherited
Metabolic Diseases, A Clinical Approach. $2^{\text {nd }}$ Edition. Springer-Verlag Berlin Heidelberg, 2017. doi. 10.1007/9783-662-49410-3.

7. Saudubray JM, Garcia-Cazorla À. Inborn errors of metabolism overview: Pathophysiology, manifestations, evaluation, and management. Pediatr Clin North Am. 2018; 65 (2): 179-208. doi.10.1016/j.pcl.2017.11.002.

8. Ibarra-González I, et al. Caracterización de errores innatos del metabolismo intermediario en pacientes mexicanos. An Pediatr. 2014; 80 (5): 310-6.

9. Guillén-López $S$, et al. Evaluación nutricional del paciente con fenilcetonuria (PKU). Acta Pediatr Mex [Internet]. 2012; 33 (6): 315-8.

10. Guillén-López $S$, et al. Conventional Phenylketonuria Treatment. J Inborn Errors Metab Screen. 2016; 4: 232640981668573.

11. Vela-Amieva $\mathrm{M}$, et al. Phenylalanine hydroxylase deficiency in Mexico: Genotype-phenotype correlations, BH4 responsiveness and evidence of a founder effect. Clin Genet. 2015; 88 (1): 62-7. https://doi.org/10.1111/cge.12444.

12. Agenda estadística, 2018. Dirección de Planeación, Instituto Nacional de Pediatría (https://www.pediatria. gob.mx/interna/planea_agestasitica.html), acceso 3 de agosto de 2020.

13. Belmont-Martínez $L$, et al. Causas de hospitalización de pacientes con errores innatos del metabolismo intermediario: análisis de una serie de casos de un hospital de tercer nivel de atención. Acta Pediatr Méx. 2020;41(3):105-14. http://dx.doi.org/10.18233/APM41No3pp105-1142065.

14. Hoogeveen IJ, et al. A preliminary study of telemedicine for patients with hepatic glycogen storage disease and their healthcare providers: from bedside to home site monitoring. J Inherit Metab Dis. 2018; 41 (6): 929-36. doi.10.1007/ s10545-018-0167-2

15. Cédula de Instrumentos Jurídicos aplicables a la práctica de la Telesalud en México. [Recurso electrónico]. México: Secretaría de Salud, Centro Nacional de Excelencia Tecnológica en Salud; 2019.

16. Secretaría de Salud, Diario Oficial de la Federación. Ley General de Salud, Secretaría de Salud, 14 de junio de 1992.

17. Cámara de Diputados, H. Congreso de la Unión. Reglamento de la Ley General de Salud en Materia de Prestación de Servicios de Atención Médica. [Online]. [Actualizado 2018; citado 2019 oct 24]. Disponible en: http://www.diputados. gob.mx/LeyesBiblio/regley/Reg_LGS_MPSAM_170718.pdf

18. Norma Oficial Mexicana NOM-004-SSA3-2012, Del expediente clínico. Diario Oficial de la Federación, 15 de octubre de 2012.

19. Tsegaye Melaku, et al. Research and intervention priorities for mental health of people living with chronic disease(s) in the midst of the COVID-19 pandemic in low resource settings: A commentary. Ann Med Surg (London) 2020. doi. 10.1016/j.amsu.2020.07.051. 
20. Fiks $A G$, et al. A defining moment for pediatric primary care telehealth. JAMA Pediatr. 2020; 10.1001/jamapediatrics.2020.1881. doi.10.1001/jamapediatrics.2020.1881

21. Amado Puentes $A$, et al. Predisposición y validación del uso de WhatsApp "como método de comunicación con familias. An Pediatr (Barc). 2020; 92 (5): 300-2. doi.10.1016/j. anpedi.2019.02.010.

22. Anderson $\mathbf{M}$, et al. Australian families living with rare disease: experiences of diagnosis, health services use and needs for psychosocial support. Orphanet J Rare Dis. 2013; 8: 22. https://doi.org/10.1186/1750-1172-8-22.

23. Ibarra-González I, et al. Caracterización de errores innatos del metabolismo intermediario en pacientes mexicanos [Characterization of inborn errors of intermediary metabolism in mexican patients]. An Pediatr (Barc). 2014; 80 (5): 310-16. doi.10.1016/j.anpedi.2013.09.003

24. Cardinali $P$, et al. The caregiving experiences of fathers and mothers of children with rare diseases in Italy: Challenges and social support perceptions. Front Psychol. 2019; 10 1780. doi.10.3389/fpsyg.2019.01780

25. Hoogeveen IJ, et al. A preliminary study of telemedicine for patients with hepatic glycogen storage disease and their healthcare providers: from bedside to home site monitoring. J Inherit Metab Dis. 2018; 41 (6): 929-36. doi.10.1007/ s10545-018-0167-2.

26. Bernstein LE, et al. Nutrition education tools used in phenylketonuria: clinician, parent and patient perspectives from three international surveys. J Hum Nutr Diet. 2014; 27 (Suppl 2):4-11. doi. 10.1111/jhn.12065.

27. García Flores EP, et al. Avances y logros del programa de tamiz metabólico neonatal en México (2012-2018). Acta Pediatr Mex. 2018; SI (39): 57S-65S. http://dx.doi. org/10.18233/APM39No6pp57S-65S1722

28. Pass KA, Thoene J, Watson MS. Emergency preparedness for newborn screening and genetic services. Genet Med. 2009; 11 (6): 455-64. doi.10.1097/GIM.0b013e3181a959c1 